\title{
Oncogenic IL7R is downregulated by histone deacetylase inhibitor in esophageal squamous cell carcinoma via modulation of acetylated FOXO1
}

\author{
MYOUNG JUN KIM ${ }^{1}$, SUNG KYUNG CHOI ${ }^{1}$, SEONG HWI HONG ${ }^{1}$, JUNG WOO EUN ${ }^{2}$, \\ SUK WOO NAM ${ }^{2}$, JEUNG-WHAN HAN ${ }^{3}$ and JUENG SOO YOU ${ }^{1}$
}

${ }^{1}$ Department of Biochemistry, Research Institute of Medical Science, School of Medicine, Konkuk University, Seoul 05029;
${ }^{2}$ Functional RNomics Research Center, College of Medicine, The Catholic University, Seoul 06591;
${ }^{3}$ Research Center for Epigenome Regulation, School of Pharmacy,
Sungkyunkwan University, Suwon 16419, Republic of Korea

Received January 1, 2018; Accepted April 18, 2018

DOI: $10.3892 /$ ijo.2018.4392

\begin{abstract}
The interleukin-7 receptor (IL7R) is generally expressed in immune cells and is critical in survival, development and homeostasis in the immune system. Advanced genome-wide cancer studies have reported that IL7R is genetically amplified in human esophageal squamous cell carcinoma (ESCC), however, the exact role of IL7R in ESCC has not been investigated. In the present study, it was found that IL7R was overexpressed in ESCC cohorts and the loss of IL7R induced anti-oncogenic effects in ESCC cell lines. A small panel of epigenetic drugs were screened for their ability to downregulate the expression of IL7R. Unexpectedly, apicidin, a histone deacetylase (HDAC) inhibitor, effectively downregulated the expression of IL7R in a dose-dependent manner at an early time-point, as determined by quantitative polymerase chain reaction and IL7R immunostaining, and did not require de novo protein synthesis. Of note, apicidin induced the acetylation of Forkhead box-containing protein, O subfamily 1, which acts as a repressor at the IL7R promoter, accompanied with depleted active histone modifications based on chromatin immunoprecipitation assay. Taken together, these results demonstrated that targeting oncogenic IL7R in ESCC by HDAC inhibitors may be a valuable therapeutic approach.
\end{abstract}

\section{Introduction}

The interleukin-7 receptor (IL7R), an important molecule in the innate and adaptive immune responses, is critical in a

Correspondence to: Dr Jueng Soo You, Department of Biochemistry, Research Institute of Medical Science, School of Medicine, Konkuk University, 120 Neungdong-ro, Gwangjin-gu, Seoul 05029, Republic of Korea

E-mail: jsyou@kku.ac.kr

Key words: interleukin-7 receptor, esophageal squamous cell carcinoma, histone deacetylase inhibitor, Forkhead box-containing protein, O subfamily 1, transcription factor acetylation variety of cellular processes, including the regulation of cell development, survival, homeostasis and differentiation of $\mathrm{T}$ cells $(1,2)$. IL7R signaling has also been reported to promote the development of $\mathrm{B}$ cells by controlling downstream signaling, including the Janus kinase/signal transducer and activator of transcription and phosphoinositide 3-kinase/AKT pathways $(3,4)$. Although the expression patterns and roles of IL7R in the immune system are well understood, its expression patterns and roles in solid tumors remain to be fully elucidated.

Esophageal cancer, the eighth most common type of cancer in the world, can be categorized into two histological types: Esophageal squamous cell carcinoma (ESCC) and esophageal adenocarcinoma (EAC) (5). Although the incidence rate of EAC has been increasing in Europe and North America, ESCC accounts for $\sim 90 \%$ of all esophageal cancer cases and is known to be more aggressive (6). For several decades, the five-year survival rate for ESCC has been low at $15-25 \%$ due to late diagnosis and induced metastasis (7). As in other types of cancer, comprehensive ESCC genome studies have been actively performed and several unexpected therapeutic targets have been identified $(8,9)$. Although the underlying mechanisms have not been fully elucidated, the findings of genome studies may improve the poor prognosis of ESCC.

As understanding of the mechanisms by which epigenetics controls gene expression patterns increases, the development of epigenetic drugs has accelerated, and certain drugs have been Food and Drug Administration-approved for clinical use $(10,11)$. The overexpression of histone deacetylases (HDAC)s and a global reduction in histone acetylation are representative epigenetic phenomena in cancer $(12,13)$. HDAC inhibitors induce histone acetylation mechanically and have been investigated extensively for activating target genes. HDAC inhibitors have a broad spectrum of targets and affect cell cycle arrest, differentiation, apoptosis, autophagy and the production of reactive oxygen species (14). The majority of mechanistic investigations of HDAC inhibitors have focused on the upregulation of tumor suppressors (15). Although the underlying mechanisms remain to be fully elucidated, few investigations on HDAC inhibitors have addressed downregulated 
genes (16). HDAC inhibitors have been reported to induce the acetylation of non-histone proteins, including transcription factors (TFs) (17,18). The post-translational modification of TFs can control localization, stability or activity. Therefore, the acetylation of TFs may provide insight into genes that are downregulated by HDAC inhibitors.

In the present study, the role of IL7R in ESCC was investigated using specific IL7R small interfering (si)RNA, and it was found that IL7R acted as an oncogene in ESCC. In a small-scale epigenetic drug screening, it was found that the HDAC inhibitor apicidin effectively suppressed the expression of IL7R. Of note, the downregulation of IL7R by apicidin was mediated by the acetylation of Forkhead box-containing protein, O subfamily 1 (FOXO1) at an early time-point and without de novo protein synthesis. Acetylated FOXO1 binds at the IL7R promoter and acts as a repressor. For the first time, to the best of our knowledge, these findings demonstrate the role of IL7R in ESCC and indicate the use of HDAC inhibitors as an IL7R regulation strategy.

\section{Materials and methods}

Cell lines. The human ESCC cell lines TE1, TE8, TE9, TE10, TE11 and ECGI were obtained from the Riken Bioresource Center Cell Bank (Tsukuba, Japan). HCE4 and HCE7 were obtained from the Korean Cell Line Bank (Seoul, Korea). Jurkat human T cell (TIB-152) were purchased from American Type Culture Collection (Manassas, VA, USA).

Cell culture and transfection. All cell lines were cultured in DMEM (Welgene, Inc., Gyeongsan, Korea; cat. no. 001-05) supplemented with $10 \%$ FBS and $1 \%$ antibiotics $(100 \mathrm{U} / \mathrm{ml}$ penicillin and $100 \mu \mathrm{g} / \mathrm{ml}$ streptomycin; Thermo Fisher Scientific, Inc., Waltham, MA, USA) at $37^{\circ} \mathrm{C}$ in an incubator containing $5 \% \mathrm{CO}_{2}$. For siRNA transfection, the cells were transfected using Lipofectamine RNAiMAX 2000 (Invitrogen; Thermo Fisher Scientific, Inc.; cat. no. 11668-019) in OPTI-MEM (Welgene, Inc.; cat. no. 004-01) according to the manufacturer's protocols and incubated for $16 \mathrm{~h}$. The Lipofectamine-containing media were replaced with standard growth media and incubated for $48 \mathrm{~h}$. Human IL7R siRNA \#1 (5'-GUAAGAAACCCAGACU GAA3') and IL7R siRNA \#2 (5'-GAGUGAAUGGAGUCCA AGU-3') was purchased from Bioneer Corporation (Daejeon, Korea). Control siRNA against GFP (5'-GACUUCCCAAGAA AUGCUU-3') was purchased from Bioneer Corporation.

Chemicals. For drug screening of a small panel of epigenetic drugs, cells were treated with the drugs at $37^{\circ} \mathrm{C}$ in an incubator containing $5 \% \mathrm{CO}_{2}$ for $24 \mathrm{~h}$. Apicidin was prepared as described in a previous study (16). DZNep was purchased from Cayman, (cat. no. 13828), BIX was from Sigma-Aldrich; EMD Millipore, Billerica, MA, USA (cat. no. SML1073), Vitamin C was from Sigma-Aldrich; EMD Millipore (cat. no. A4403) and C646 was from EMD Millipore (cat. no. 382113$)$.

RNA extraction and reverse transcription-quantitative polymerase chain reaction $(R T-q P C R)$ analysis. Total RNA was extracted using TRIzol reagent. The RNA samples were adjusted to the same concentration and reverse transcribed using a
High-Capacity cDNA reverse transcription kit (Applied Biosystems; Thermo Fisher Scientific, Inc.; cat. no. 4368813). Synthesized cDNA was performed using a Cycler $^{\circledR} 480$ II with LightCycler ${ }^{\circledR}$ 480 SYBR Green I master mix (Roche Diagnostics, Basel, Switzerland; cat. no. 04887352001), according to the manufacturer's protocols. The RT-qPCR primer sequences used were as follows: IL7R, forward, 5'-GGAGAAAGTGGCTATGC TCAAAA-3' and reverse, 5'-CCATTCACTTCCAACTGGC TAT-3'; P21, forward, 5'-GAGGCCGGGATGAGTTGGGAG GAG-3' and reverse, 5'-CAGCCGGCGTTTGGAGTGGTA GAA-3'; GAPDH, forward, 5'-GAGTCAACGGATTTGGT CGT-3' and reverse, 5'-TGGAAGATGGTGATGGGATT-3'. The RT-qPCR analysis was performed with an initial denaturation step of $5 \mathrm{~min}$ at $95^{\circ} \mathrm{C}$, followed by 45 cycles at $95^{\circ} \mathrm{C}$ for $10 \mathrm{sec}, 60^{\circ} \mathrm{C}$ for $10 \mathrm{sec}$, and $72^{\circ} \mathrm{C}$ for $10 \mathrm{sec}$. Quantification of RNA values was determined automatically using the LightCycler ${ }^{\circledR} 480$ program (Roche Diagnostics).

Western blot analysis. The cells were lysed in RIPA buffer containing protease and phosphatase inhibitors. The lysates were centrifuged at $15,000 \times \mathrm{g}$ for $15 \mathrm{~min}$ at $4^{\circ} \mathrm{C}$ and the supernatants were transferred to new tubes. Proteins were quantified using the Bradford protein assay, and lysates were diluted as appropriate to ensure the equal loading of total protein. Depending on the experiment, a volume containing 40-80 $\mu$ g cell lysates from each sample was boiled in sample buffer [60 mM Tris- $\mathrm{HCl}$ (pH 6.8), 25\% glycerol, $14.4 \mathrm{mM}$ 2-mercaptoethanol and $1 \%$ bromophenol blue]. The lysates were then resolved by SDS-PAGE on $12 \%$ gels and transferred onto nitrocellulose membranes. The membranes were incubated with $5 \%$ skim milk for $1 \mathrm{~h}$ at room temperature and incubated with anti-Ac-FOXO1 (Santa Cruz Biotechnology, Inc., Dallas, TX, USA; cat no. sc49437, 1:100), anti-phosphorylated histone H3 serine 10 (pH3ser10; EMD Millipore; cat. no. 06-570, 1:1,000), H3 (Abcam, Cambridge, UK; cat. no. ab1791, 1:2,000), or $\beta$-actin (Cell Signaling Technology, Inc., Danvers, MA, USA; cat. no. 4967, 1:2,000) overnight at $4^{\circ} \mathrm{C}$. The membranes were washed with TBS-T and probed with an HRP-conjugated secondary antibody (Cell signaling Technology, Inc.; cat. no. 7074, 1:5,000) in 5\% skim milk for $1 \mathrm{~h}$ at room temperature. Following washing with Trisbuffered saline containing $0.5 \%$ Tween, the membranes were visualized using an enhanced chemiluminescence detection kit (LF-QC0103 AB Frotier) following exposure on an LAS-3000 image detection system (Fujifilm, Tokyo, Japan).

Immunocytochemistry. The cells were seeded on poly-L-lysinecoated glass coverslips in standard growth media for $16 \mathrm{~h}$. The cells were washed with cold PBS and fixed with $4 \%$ paraformaldehyde for $30 \mathrm{~min}$ at room temperature. The fixed cells were permeabilized with $0.1 \%$ Triton X-100 for 5 min and blocked in $3 \%$ goat serum (Jackson ImmunoResearch Laboratories, Inc., West Grove, PA, USA; cat. no. 005-000-121) for $30 \mathrm{~min}$. The cells were stained with Ac-FOXO1 (Santa Cruz Biotechnology, Inc.; cat. no. sc49437, 1:50) and Alexa Fluor ${ }^{\circledR}$ anti-rabbit 488 secondary antibodies (Jackson ImmunoResearch, Inc.; cat. no. 111-545-003, 1:500) for $30 \mathrm{~min}$ at room temperature. The nuclei were counterstained with DAPI. The fluorescent signals were detected, and images were captured using a fluorescence microscope. 
Cell wound-healing assay. The cells transfected with IL7R siRNA were seeded into 6-well plates and incubated in $5 \% \mathrm{CO}_{2}$ at $37^{\circ} \mathrm{C}$ for $16 \mathrm{~h}$. When the cells reached confluence, a wound was scratched using a pipette tip, fresh culture media was added, and the cells were incubated in $5 \% \mathrm{CO}_{2}$ at $37^{\circ} \mathrm{C}$. Images of the scratched areas were captured under a microscope (Olympus IX71; Olympus Corporation, Tokyo, Japan) at 0,12 and $24 \mathrm{~h}$ post-wounding.

Cell cycle analysis. Cell cycle assays were performed using the Cycletest Plus DNA reagent kit (BD Biosciences, Franklin Lakes, NJ, USA; cat. no. 340242), according to the manufacturer's protocol. The cell cycle profiles were analyzed using a FACScan flow cytometer (BD Biosciences).

$I L 7 R^{+}$cell staining. The cells were washed twice with cold PBS, centrifuged at $1,000 \times \mathrm{g}$ for $5 \mathrm{~min}$ at $4^{\circ} \mathrm{C}$, resuspended in FACS buffer containing IL7R antibodies (eBioscience, Inc., San Diego, CA, USA; cat. no. 17-1278-42) and IgGк (eBioscience, Inc.; cat. no. 17-4714-42), and incubated for $1 \mathrm{~h}$ at $4^{\circ} \mathrm{C}$. Following washing, fixation buffer was added, and the cells were incubated for $20 \mathrm{~min}$ at $4^{\circ} \mathrm{C}$. The cells were washed with cold PBS and resuspended in $4 \%$ paraformaldehyde. The cells were analyzed using a FACScan flow cytometer (BD Biosciences).

Chromatin immunoprecipitation (ChIP) assay. ChIP assays were performed according to the manufacturer's protocol (Upstate Biotechnology, Inc., Lake Placid, NY, USA). DNA was sheared by sonication to achieve fragments between 200 and 500 bp. The sheared DNA was pre-cleared with protein A magnetic beads (EMD Millipore; cat. no. 92590), and $50 \mu \mathrm{g}$ DNA was then immunoprecipitated by Ac-FOXO1 (Santa Cruz Biotechnology, Inc.; cat. no. sc49437) or AcH3 (EMD Millipore; cat. no. 06-866). Following IP, the recovered chromatin fragments were subjected to PCR analysis. IgG control experiments were performed for all ChIPs and incorporated into the IP/Input (1\%) by presenting the results as (IP-IgG)/(Input-IgG). The PCR primer sequences used were as follows: IL7R proximal promoter(pp)1,forward,5'-CAGGGAATATCCAGGAGGAA-3' and reverse, 5'-AGGGAGGGAGGAAGACAGAG-3'; IL7R pp2, forward, 5'-TTGGAAGTTGGAGGTAAGCTATTT-3' and reverse, 5'-TGCTAGGACTACAGGCATGA-3'; P21pp, forward, 5-GACTGGGGGAGGAGGGAAG-3' and reverse,5'-CTTCGG CAGCTGCTCACAC-3'.

Use of datasets. The human ESCC cohorts datasets (GSE23400 and GSE29001) from the National Center for Biotechnology Information (NCBI) Gene Expression Omnibus (GEO) database (www.ncbi.nlm.nih.gov/geo/) were used, and the GSE80005 mouse ESCC experimental dataset was used.

Statistical analysis. The results are expressed as the mean \pm standard error of the mean. The majority of statistical comparisons were calculated using one-way analysis of variance followed by Bonferroni's post hoc test using GraphPad Prism software version 5 (GraphPad Software, Inc., La Jolla, CA, USA). $\mathrm{P}<0.05$ was considered to indicate a statistically significant difference.

\section{Results}

$I L 7 R$ is overexpressed and the repression of IL7R induces antioncogenic effects in ESCC cell lines. IL7R has been reported to be genetically amplified in ESCC, however, the expression patterns and roles of IL7R in ESCC have not been reported (9). In the present study, the expression of IL7R was first compared in publicly available datasets of human ESCC cohorts from the NCBI GEO database (GSE23400 and GSE29001). IL7R was overexpressed in ESCC tissue samples compared with normal tissue samples in cohorts (Fig. 1A). In the animal model data, a significantly higher expression of IL7R was observed between the normal esophagus and ESCC mice (Fig. 1B). Subsequently, the expression of IL7R was examined by RT-qPCR analysis to screen for the mRNA expression of IL7R in various ESCC cell lines compared with the Jurkat human $\mathrm{T}$ lymphocyte cell line, which is known to express IL7R (19). The majority of the ESCC cell lines showed comparable expression of IL7R to the Jurkat cells (Fig. 1C). The HCE7 cells showed distinct expression of IL7R among the ESCC cell lines screened (Fig. 1C); therefore, subsequent experiments were performed mainly using the HCE7 cell line.

To investigate whether the expression of IL7R induces oncogenic effects in ESCC, siRNAs were used to knockdown the expression of IL7R in HCE7 and HCE4 cells. The IL7R-specific siRNAs effectively repressed the expression of IL7R at the mRNA and protein levels (Fig. 1D and E). IL7R knockdown induced mild G2 cell-cycle arrest in the HCE7 and HCE4 cells, and increased the level of P21 (Fig. 1F and G). Furthermore, it was observed that the knockdown of IL7R in HCE7 and HCE4 cells reduced the wound-healing capacity (Fig. 1H). Taken together, the results demonstrated that IL7R was overexpressed and may have an oncogenic role in ESCC.

In addition to ESCC, it was observed that IL7R was overexpressed in EACs and the high expression of IL7R was correlated with poor prognosis in terms of disease-free survival rate $(\mathrm{P}=0.042$, data not shown).

IL7R is downregulated by apicidin in a dose- and timedependent manner in HCE7 cells. To determine a strategy for IL7R regulation, a small panel of drugs that modulate histone acetylation or methylation were screened. Among the small molecules, apicidin $(14,20)$, an HDAC inhibitor, induced marked downregulation of the expression of IL7R in HCE7 cells compared with other drugs (Fig. 2A). To investigate whether IL7R is directly targeted by apicidin, HCE7 and HCE4 cells were exposed to $0.1,0.5,1$ or $5 \mu \mathrm{M}$ apicidin for $24 \mathrm{~h}$. The mRNA levels of IL7R were markedly decreased in the two cell lines (Fig. 2B). Apicidin has been reported to induce G1 cell-cycle arrest $(14,16)$, but in the present study, apicidin induced $\mathrm{G} 2$ cell-cycle arrest in a dose-dependent manner in HCE7 and HCE4 cells, similar to the loss of IL7R (Fig. 2C). The downregulated the expression of IL7R by apicidin was observed at an early time-point, from $3 \mathrm{~h}$, and $>95 \%$ protein was depleted at $12 \mathrm{~h}$ in the HCE7 cells (Fig. 2D and E). These time-points corresponded with cell-cycle arrest and the downregulation of the mitotic marker pH3Ser10 $(21,22)$ (Fig. 2F and G). Following treatment with apicidin for $24 \mathrm{~h}$, the mRNA and protein levels of IL7R were also downregulated 
A

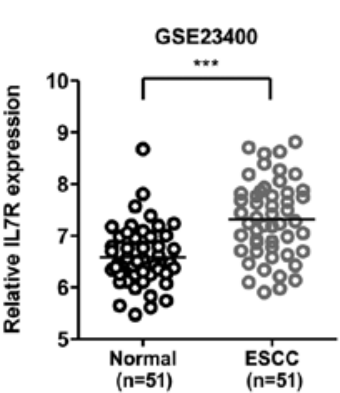

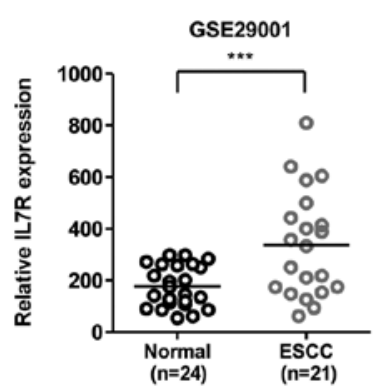
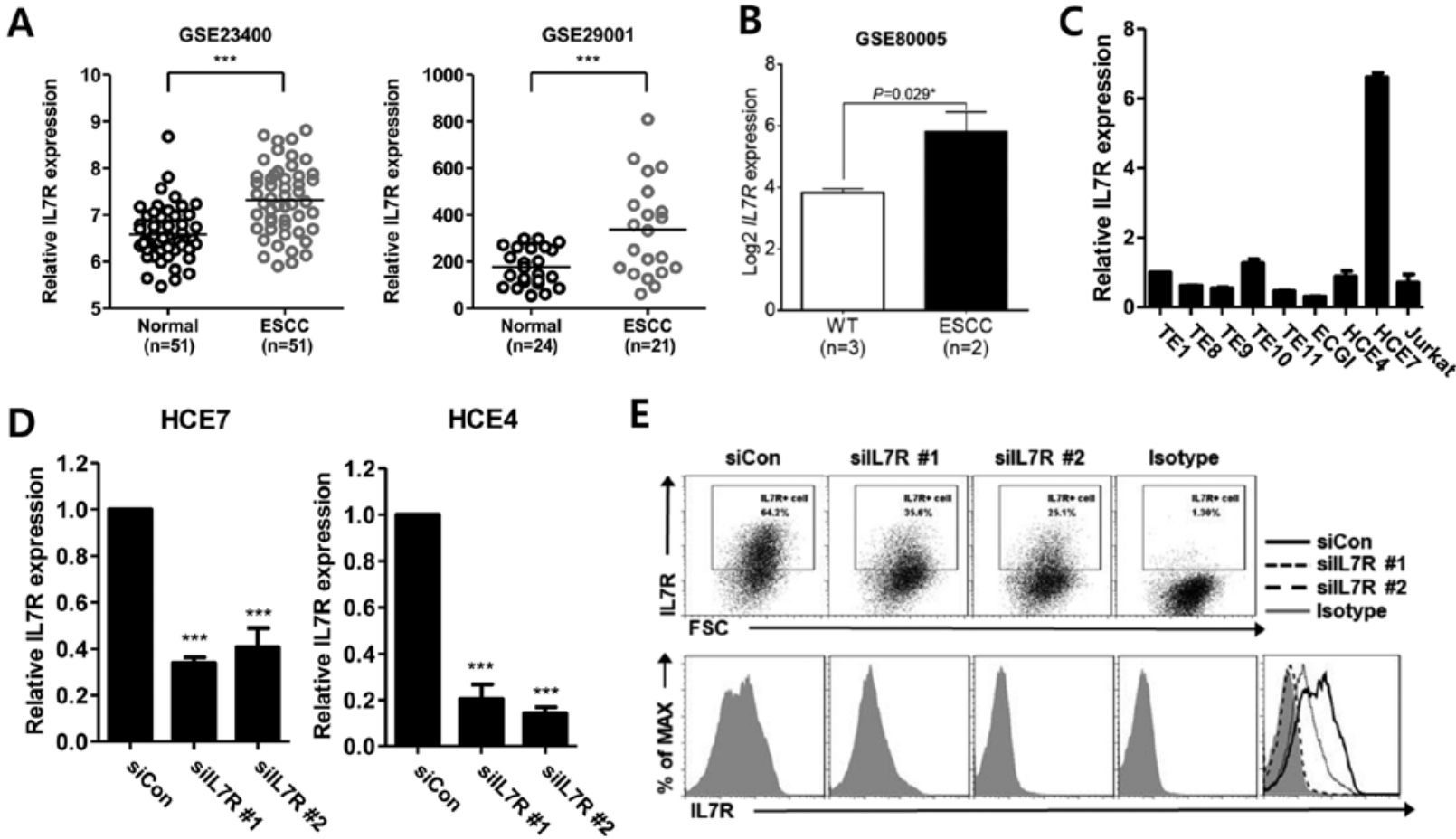

$\mathbf{E}$

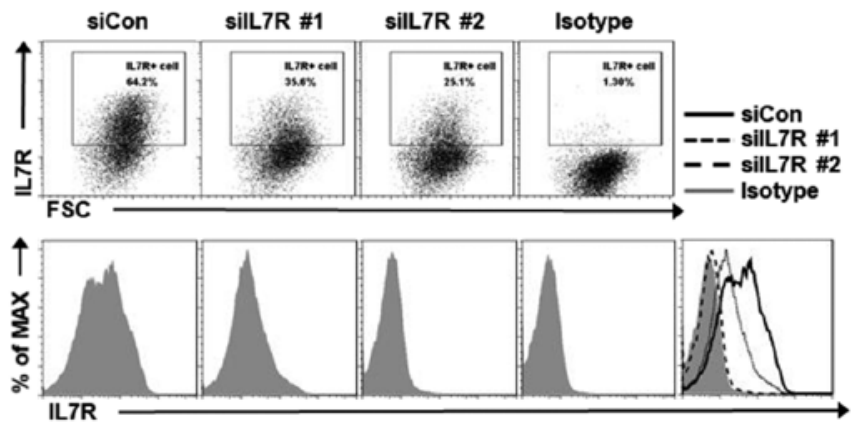

$\mathbf{F}$

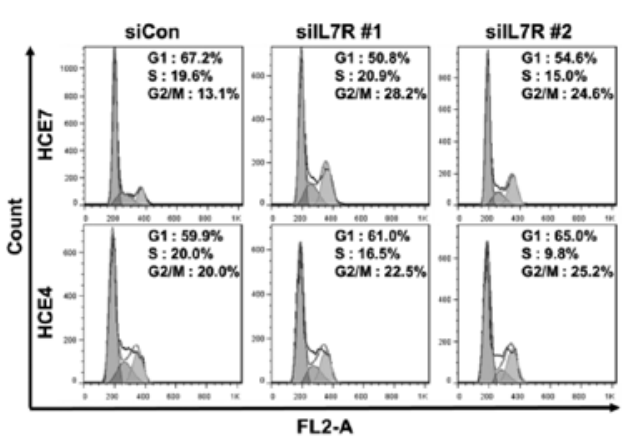

H
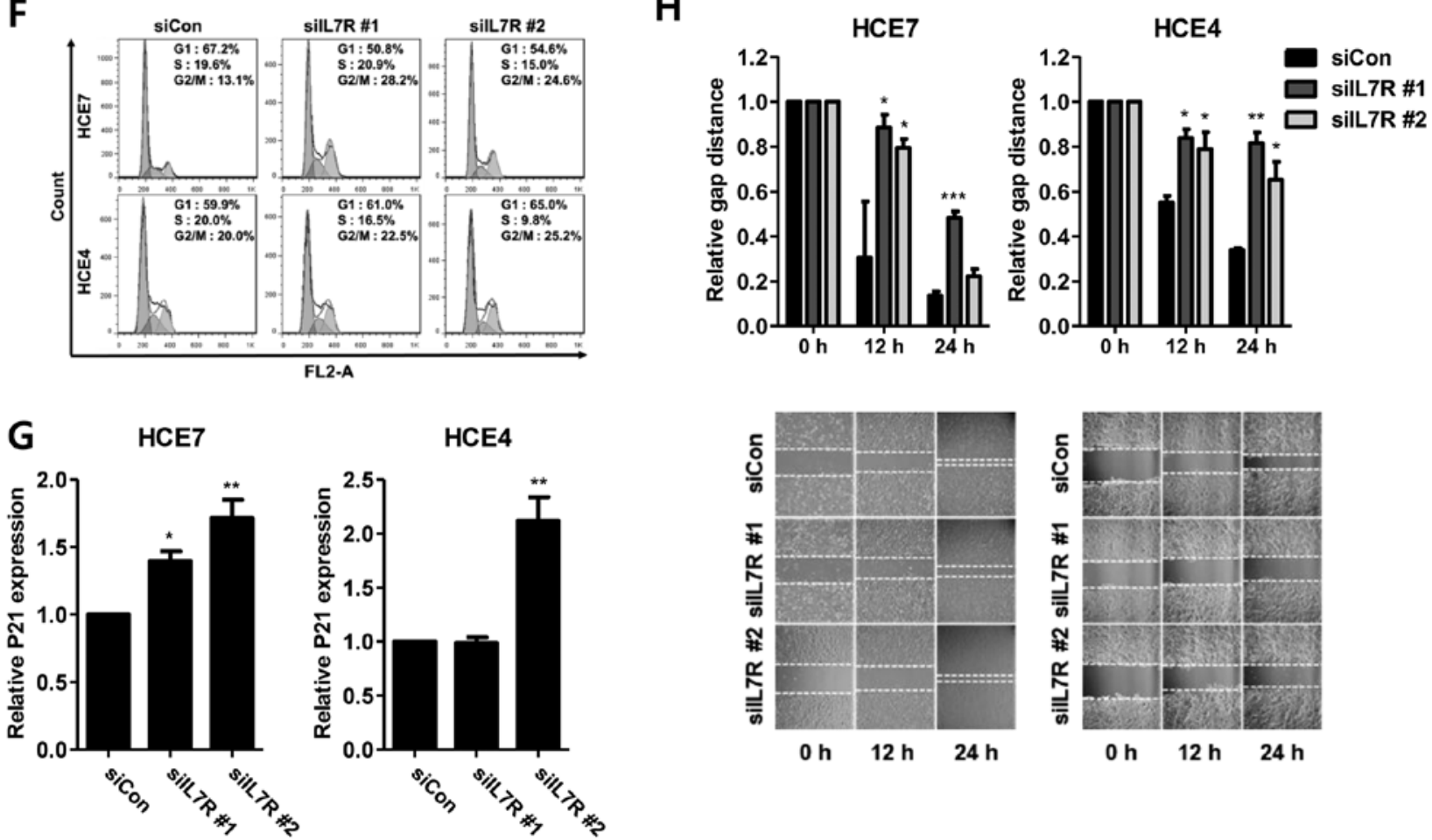

Figure 1. Downregulation of IL7R induces anti-oncogenic effects in ESCC cells. (A) Data sets GSE23400 and GSE29001 were obtained from the National Center for Biotechnology Information Gene Expression Omnibus database. ${ }^{* * *} \mathrm{P}<0.001$ vs. normal tissue. (B) IL7R expression data were obtained from animal dataset GSE80005. (C) mRNA expression levels of IL7R were screened by RT-qPCR analysis in ESCC cell lines and compared with Jurkat cells. Transfection of HCE7 and HCE4 cells with IL7R-targeted and control siRNA were performed. After $72 \mathrm{~h}$, the expression of IL7R was determined by (D) RT-qPCR analysis and (E) flow cytometry. The $(\mathrm{F})$ cell cycle, $(\mathrm{G})$ expression of $\mathrm{P} 21(\mathrm{G}$ and $\mathrm{H})$ wound-healing capacity were also determined. ${ }^{*} \mathrm{P}<0.05$, ${ }^{* *} \mathrm{P}<0.01$ and ${ }^{* * *} \mathrm{P}<0.001$ vs. control. ESCC, esophageal squamous cell carcinoma; IL7R, interleukin-7 receptor; RT-qPCR, reverse transcription-quantitative polymerase chain reaction; siRNA/si, small interfering RNA; WT, wild-type; Con, control.

in Jurkat cells (Fig. 2H and I), suggesting that the effects of apicidin on IL7R are not specific to ESCC.

De novo protein synthesis is not required for repression of the expression of IL7R by apicidin. The present study also investigated whether the downregulation in the expression of IL7R by apicidin required de novo protein synthesis. As shown in Fig. 3, pretreatment of the HCE7 cells with the protein synthesis inhibitor cycloheximide did not rescue the effects of apicidin on the mRNA expression of IL7R. These 
A

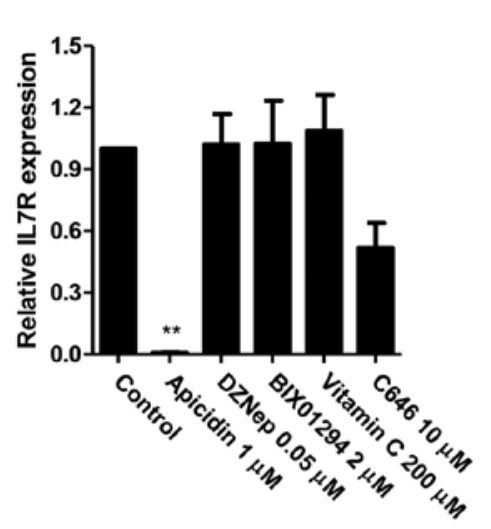

B

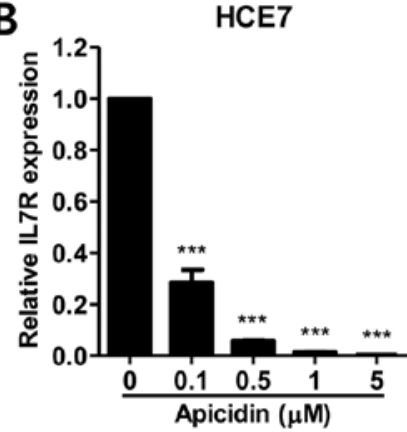

HCE4

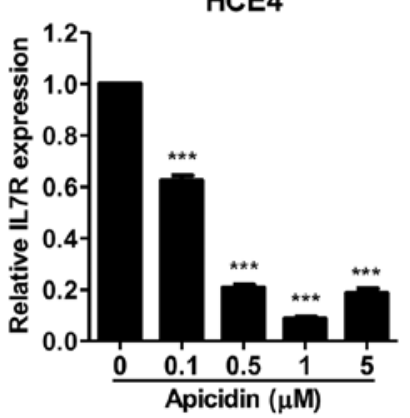

C

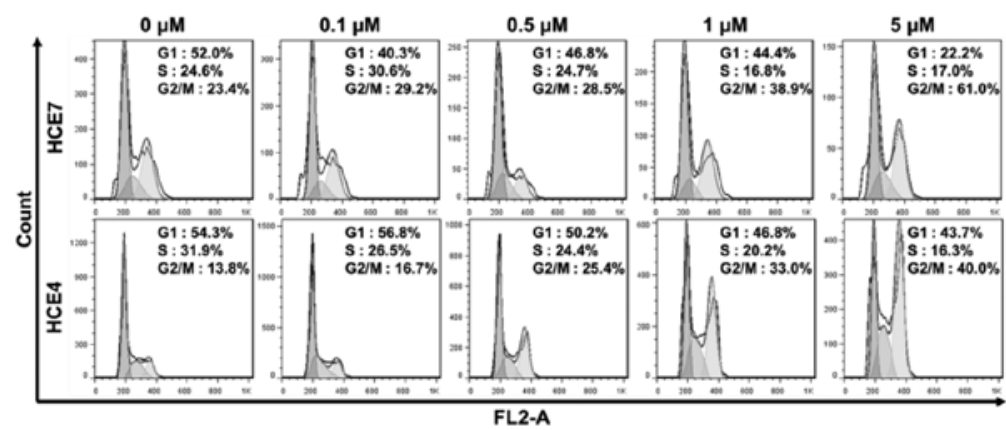

D

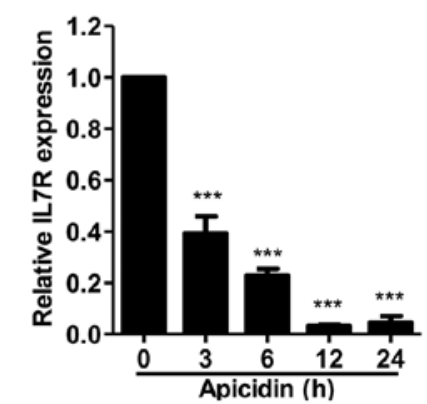

E

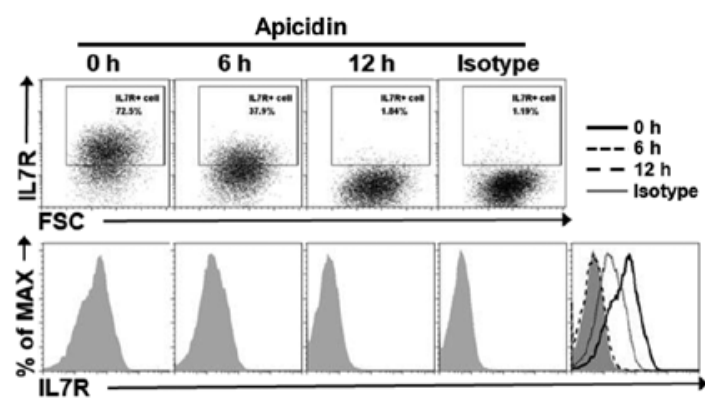

$\mathbf{F}$

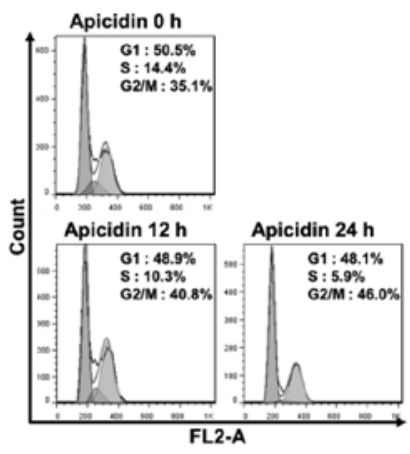

G

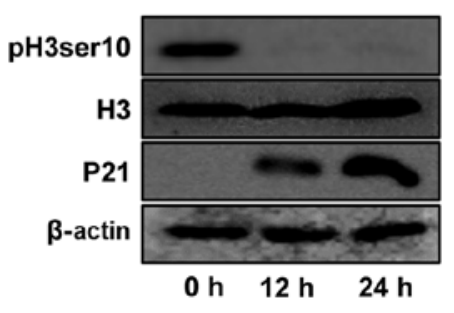

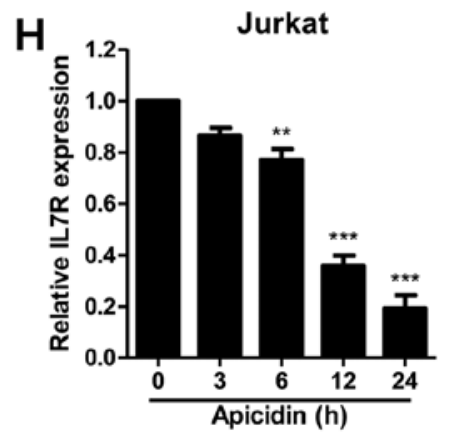

I

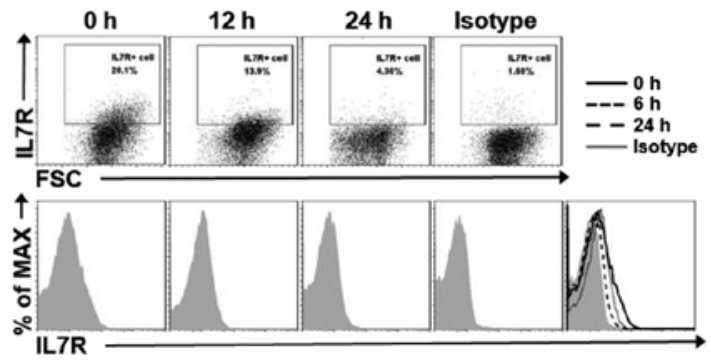

Figure 2. Apicidin induces the downregulation of IL7R and exhibits anti-oncogenic effects in HCE7 cells. (A) Small molecules were used to screen the expression of IL7R in HCE7 cells. Expression of IL7R was determined by reverse transcription-quantitative polymerase chain reaction analysis at 24 h post-drug treatments. (B) mRNA level of IL7R and (C) cell cycle were determined in HCE7 and HCE4 cells treated with increasing concentrations of apicidin for 24 h. HCE7 cells were treated with $1 \mu \mathrm{M}$ apicidin for the indicated time-points. (D) mRNA and (E) protein levels of IL7R were determined. (F) Cell cycle and (G) protein levels of pH3Ser10 and P21 were determined. Jurkat cells were treated with $1 \mu \mathrm{M}$ apicidin for the indicated time-points. (H) mRNA and (I) protein levels of IL7R were determined. ${ }^{* *} \mathrm{P}<0.01$ and ${ }^{* * *} \mathrm{P}<0.001$ vs. control. IL7R, interleukin-7 receptor; pH3Ser10, phosphorylated histone H3 Serine 10 .

data suggested that the HDAC inhibitor apicidin suppressed IL7R independent of de novo protein synthesis.

Apicidin-induced acetylation of FOXO1 acts as a repressor of the expression of IL7R. To understand the mechanisms underlying the downregulation of IL7R by apicidin, the present study investigated the upstream regulators of IL7R. To date, zinc finger E-box binding homeobox 2 (ZEB2), GATA binding protein 3 (GATA3) and FOXO1 have been reported to regulate the transcription of IL7R (23-27). The 
A

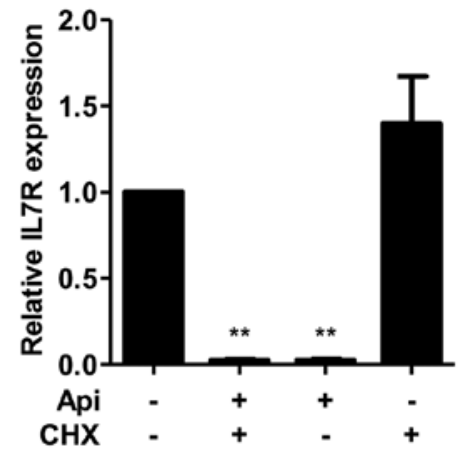

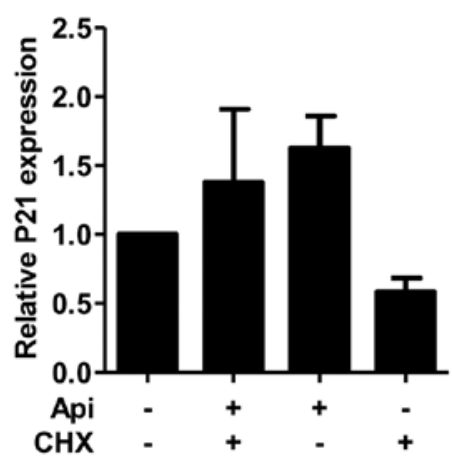

\section{B}

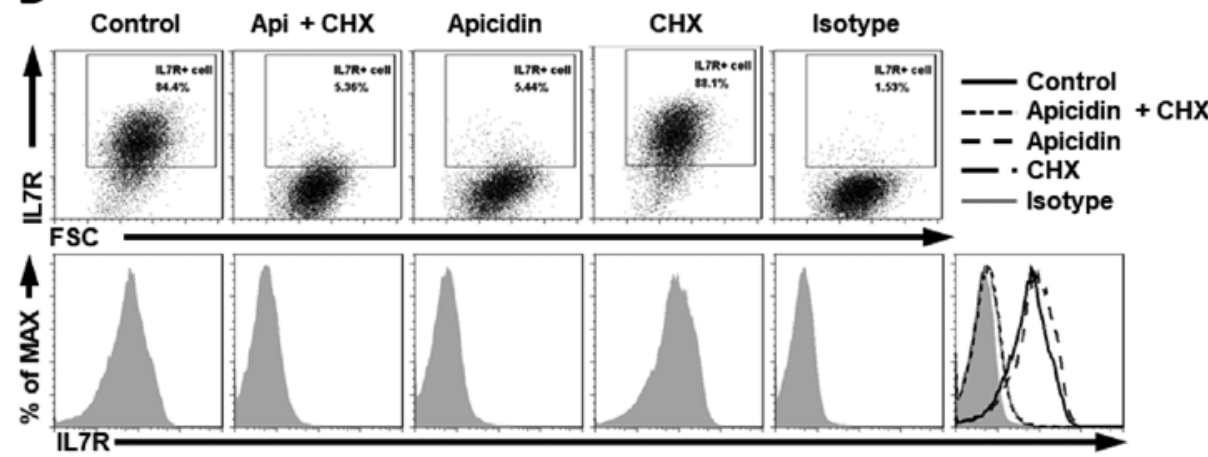

Figure 3. De novo protein synthesis is not required for the transcriptional repression of IL7R by apicidin. HCE7 cells were pretreated with CXH for $1 \mathrm{~h}$ and then treated with apicidin for $24 \mathrm{~h}$. (A) mRNA levels of IL7R (left panel) and P21 (right panel) were determined by reverse transcription-quantitative polymerase chain reaction. ${ }^{* *} \mathrm{P}<0.01$ vs. control. (B) Protein levels of IL7R were determined by flow cytometry. IL7R, interleukin-7 receptor; CXH, cycloheximide; Api, apicidin.

present study investigated the putative binding sites of these TFs based on a consensus sequence of the IL7R upstream region covering $-5 \mathrm{~kb}$ from the transcription start site (TSS). There are several binding motifs for the three TFs (Fig. 4A). Subsequently, a literature search was performed for the posttranslational acetylation of the TFs. Whereas FOXO1 has eight sites for acetylation, ZEB2 does not have a putative site and GATA3 has only one site. Of note, FOXO1 has been known to translocate between the cytoplasm and nucleus dependent on post-translational modifications, including phosphorylation and acetylation (28). Therefore, the present study focused on FOXO1 and hypothesized that apicidin induces the acetylation of FOXO1, and that acetylated FOXO1 translocates to the nucleus and affects the transcription of IL7R. To test this hypothesis, western blot and immunocytochemical analyses of acetylated FOXO1 were performed, using acetylated FOXO1 antibody for the detection of three residues following apicidin treatment. An increase in acetylated FOXO1 was observed as early as $15 \mathrm{~min}$ and accumulated in the nucleus up to $6 \mathrm{~h}$ (Fig. 4B and C). Subsequently, a ChIP assay was used to examine whether there is a change in the recruitment of acetylated FOXO1 to the IL7R DNA regulatory regions. As shown in Fig. 4A, primers were designed for two IL7R promoters, one bearing the FOXO1 binding motif (ppl) and the other covering the TSS (pp2). The binding of acetylated FOXO1 was significantly increased at both promoters accompanied by a decrease in acetylated histone H3 (Fig. 4D), suggesting that acetylated FOXO1 may act as a repressor of the transcription of IL7R. As a control, a ChIP assay was performed for the P21pp. There was a decrease in acetylated FOXO1-binding, however, the increase in acetylated histone $\mathrm{H} 3$ was statistically significant at the P21 promoter at $6 \mathrm{~h}$ not $3 \mathrm{~h}$, respectively (Fig. 4E). It is possible that the increased level of acetylated FOXO1 by apicidin results in increased binding at the IL7R promoter, however, based on the decrease of acetylated FOXO1 at the P21 promoter, the increased enrichment of acetylated FOXO1 at the IL7R promoter is specific, rather than simply an increased level of acetylated FOXO1. Taken together, the results indicated that apicidin treatment induces acetylated FOXO1 to translocate from the cytoplasm to the nucleus where it bound to the IL7R promoter and repressed transcription.

\section{Discussion}

In the present study, the role of IL7R in ESCC was investigated. It was found that acetylated FOXO1 is a critical repressor of the expression of IL7R in ESCC. Data indicating that IL7R was overexpressed in multiple datasets further supports previous reports of IL7R genetic amplification in ESCC. The knockdown of IL7R induced antitumorigenic effects in ESCC cell lines. The HDAC inhibitor, apicidin, suppressed the transcription of IL7R by inducing the translocation of acetylated FOXO1 to the nucleus independent of de novo protein synthesis. Anti-acetylated FOXO1 ChIP assays demonstrated that acetylated FOXO1 acts as a critical repressor of IL7R under apicidin treatment. Taken together, the present study demonstrated that IL7R may be an oncogene in ESCC and that HDAC inhibitors may be an effective therapeutic strategy in ESCC by modulating acetylated FOXO1, a key repressor of the transcription of IL7R. 
A

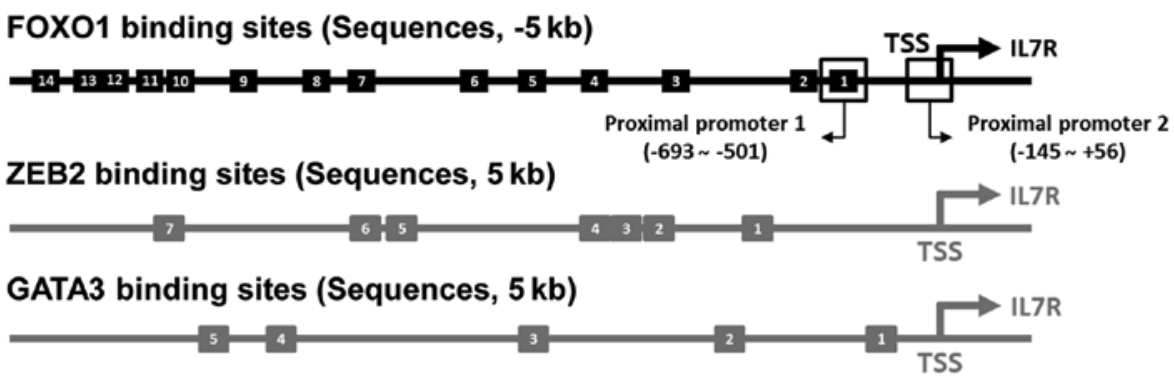

B

C

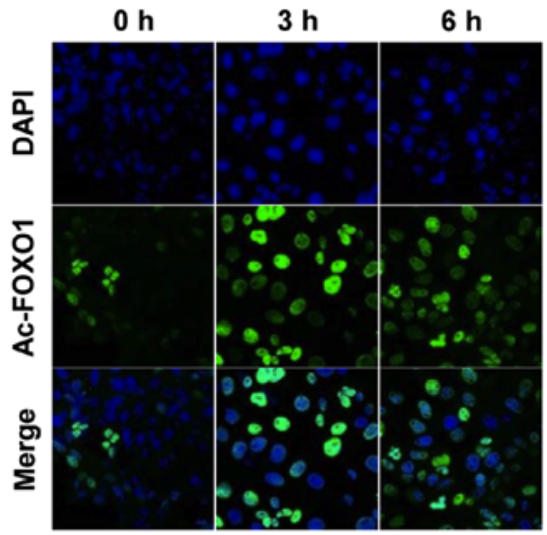

D
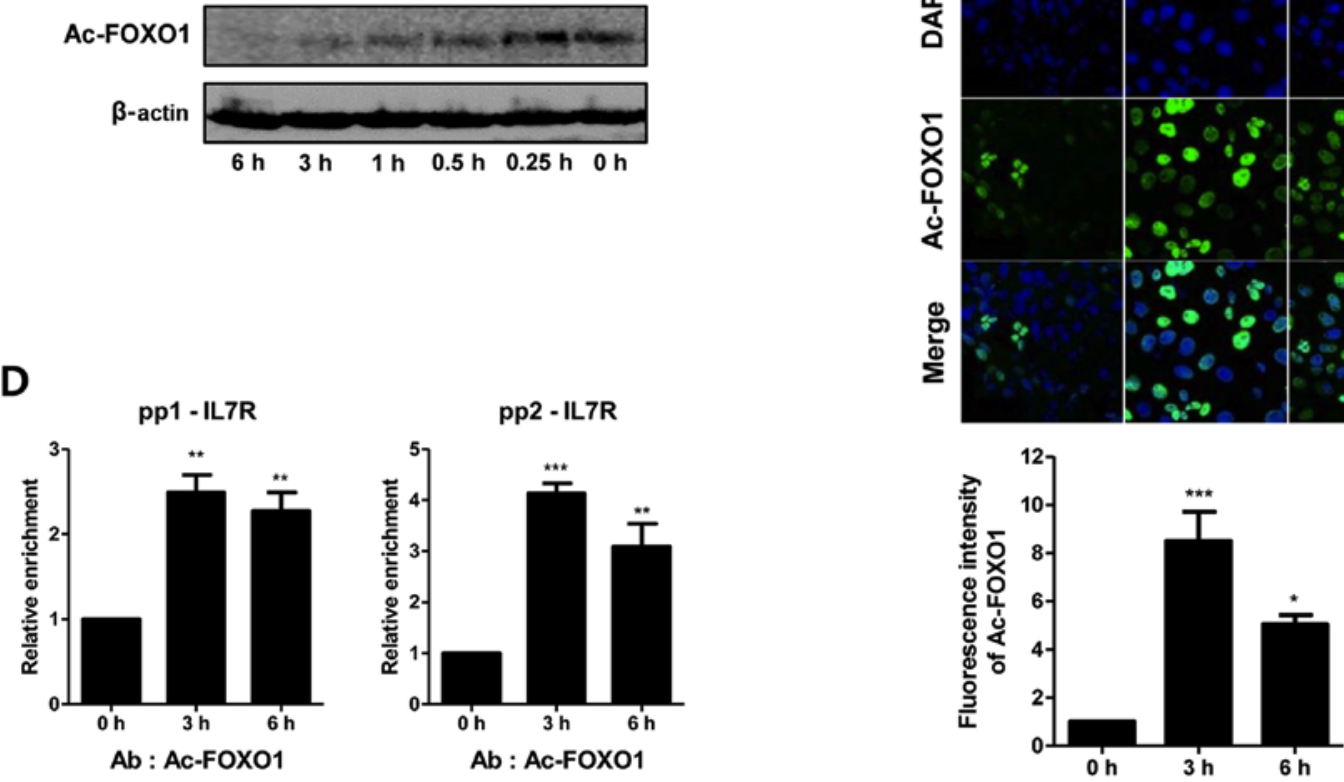

pp1 - IL7R

pp2 - IL7R
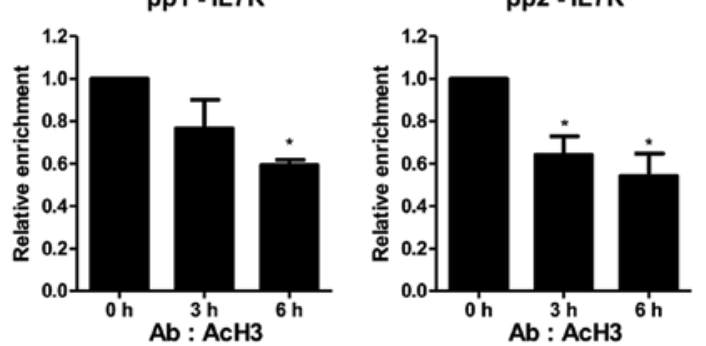

$\mathbf{E}$

pp - P21

pp - P21
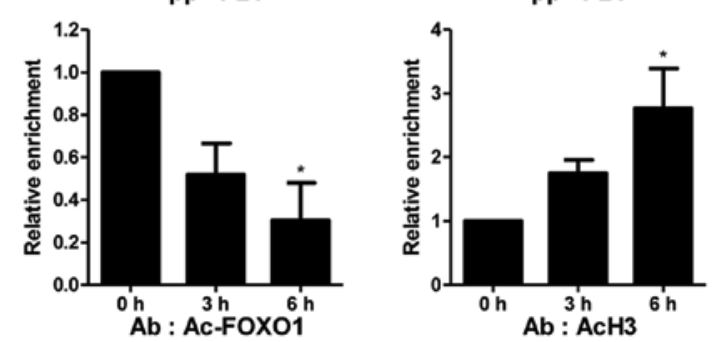

Figure 4. Acetylated FOXO1 induced by apicidin binds to promoters of IL7R and acts as a repressor. (A) Schematic diagram of FOXO1, ZEB2 and GATA3 binding sites at the promoters of IL7R up to $-5 \mathrm{~kb}$. Acetylated FOXO1 induced by apicidin was confirmed by (B) western blot analysis and (C) immunocytochemistry. Chromatin was immunoprecipitated with anti-AcH3 and anti-Ac-FOXO1 at the (D) two IL7R proximal promoters (pp1 and pp2) and (E) P21pp. ${ }^{*} \mathrm{P}<0.05,{ }^{* *} \mathrm{P}<0.01$ and $^{* * * *} \mathrm{P}<0.001$ vs. control. IL7R, interleukin-7 receptor 1; FOXO1, Forkhead box-containing protein, O subfamily 1; ZEB2, zinc finger E-box binding homeobox 2; GATA3, GATA binding protein 3; TSS, transcription start site; pp, proximal promoter; Ab, antibody; Ac, acetylated.

One benefit of whole exome/genome sequencing of human cancer is identifying unexpected therapeutic targets $(29,30)$. Although the expression patterns and roles of IL7R in the immune system have been well established, the presence of IL7R in stromal cells, particularly in ESCC, is not well understood. Although a pioneer study showed that IL7R is amplified in ESCC (9), the actual expression patterns and roles of IL7R have not been investigated previously. The present study showed that IL7R was overexpressed in cohorts with ESCC compared with normal tissue and is involved as a critical oncogene in ESCC cell lines, determined using loss of function experiments (Fig. 1). In the process of identifying a strategy to regulate the expression of IL7R, it was found that the HDAC inhibitor, apicidin, downregulated the expression of IL7R at an early time-point and, unexpectedly, independently of de novo protein synthesis (Figs. 2 and 3). To date, the majority of studies have reported that the effects of HDAC inhibitors are mediated by the upregulation of target genes by the increase of acetylated histones at the DNA regulatory regions of target genes, which is expected due to HDACs eliminating histone acetylation, an active mark of transcription. In the present study, FOXO1 acetylation was presumably a primary event of the HDAC inhibitor apicidin for the downregulation of IL7R via changing FOXO1 localization and DNA binding affinity, accompanied with the local decrease of acetylated $\mathrm{H} 3$ at the IL7R promoter. The results of the present study suggested that 
HDAC inhibitors downregulate genes in a non-histone proteindependent manner, by modulating the acetylation of TFs, a finding beneficial to future investigations of the mechanism.

The FOXO family (FOXO1, FOXO3a, FOXO4 and FOXO6) includes well-established TFs with dual activities in a wide range of functions, including metabolism, cell death, cell cycle and oxidative stress responses (31-34). The activity of FOXO is regulated by protein expression levels, subcellular localization and post-translational modifications (35). The most common post-translational modifications of FOXO are phosphorylation, acetylation, and ubiquitination, and TF activity is dependent upon these modifications; however, one modification does not always control the direction for its activity. For example, phosphorylation by AKT induces accumulation in the cytosol to disrupt interactions with DNA (36), whereas phosphorylation by mammalian Ste20-like kinase1 and c-Jun kinase 1 in response to oxidative stress allows translocation to the nucleus (37-39). The acetylation of FOXO also affects subcellular localization, DNA binding capacity and sensitivity of phosphorylation $(40,41)$. In the present study, it was found that apicidin induced the acetylation of FOXO1 in the nucleus and increased the binding of acetylated FOXO1 at the IL7R promoter, with a decrease in active histone modifications (Fig. 4). It is worth determining whether the apicidin-induced acetylation of FOXO1 has effects on other modifications, including phosphorylation, and investigating the mechanisms by which acetylated FOXO1 acts as a repressor.

In the present study, it was demonstrated that the overexpression of IL7R had a tumorigenic role in ESCC and suggested an HDAC inhibitor strategy to regulate the expression of IL7R. Apicidin, the HDAC inhibitor, regulated IL7R via the modulation of acetylated FOXO1. The results of the present study suggest that genome-wide cancer studies lead to an improved understanding of newly identified therapeutic targets, allowing the development of regulation strategies for treating intractable forms of cancer, including ESCC.

\section{Acknowledgements}

We are grateful to Dr Paul S. Mischel (University of California, San Diego, CA, USA) for the FOXO1-related discussion.

\section{Funding}

This study was supported by the Basic Science Research Program through the National Research Foundation of Korea funded by the Ministry of Science, ICT \& Future Planning (grant nos. 2016R1C1B3007534 and 2016R1A5A2012284).

\section{Availability of data and materials}

The datasets used and/or analyzed during the current study are available from the corresponding author on reasonable request.

\section{Authors' contributions}

JSY conceived and designed the experiments. MJK, SKC and SHH performed the experiments. MJK, JWE, SWN and JWH analyzed the data. MJK and JSY wrote the study.

\section{Ethics approval and consent to participate}

Not applicable.

\section{Consent for publication}

Not applicable.

\section{Competing interests}

The authors declare that they have no competing interests.

\section{References}

1. Peschon JJ, Morrissey PJ, Grabstein KH, Ramsdell FJ, Maraskovsky E, Gliniak BC, Park LS, Ziegler SF, Williams DE, Ware CB, et al: Early lymphocyte expansion is severely impaired in interleukin 7 receptor-deficient mice. J Exp Med 180: 1955-1960, 1994.

2. von Freeden-Jeffry U, Vieira P, Lucian LA, McNeil T, Burdach SE and Murray R: Lymphopenia in interleukin (IL)-7 gene-deleted mice identifies IL-7 as a nonredundant cytokine. J Exp Med 181: 1519-1526, 1995

3. Goetz CA, Harmon IR, O'Neil JJ, Burchill MA and Farrar MA: STAT5 activation underlies IL7 receptor-dependent B cell development. J Immunol 172: 4770-4778, 2004.

4. Jiang Q, Li WQ, Aiello FB, Mazzucchelli R, Asefa B, Khaled AR and Durum SK: Cell biology of IL-7, a key lymphotrophin. Cytokine Growth Factor Rev 16: 513-533, 2005.

5. Zhang XM and Guo MZ: The value of epigenetic markers in esophageal cancer. Front Med China 4: 378-384, 2010

6. Rustgi AK and El-Serag HB: Esophageal carcinoma. N Engl J Med 371: 2499-2509, 2014.

7. Pennathur A, Gibson MK, Jobe BA and Luketich JD: Oesophageal carcinoma. Lancet 381: 400-412, 2013.

8. Lin DC, Hao JJ, Nagata Y, Xu L, Shang L, Meng X, Sato Y, Okuno Y, Varela AM, Ding LW, et al: Genomic and molecular characterization of esophageal squamous cell carcinoma. Nat Genet 46: 467-473, 2014.

9. Song Y, Li L, Ou Y, Gao Z, Li E, Li X, Zhang W, Wang J, Xu L, Zhou Y, et al: Identification of genomic alterations in oesophageal squamous cell cancer. Nature 509: 91-95, 2014.

10. You JS and Han JH: Targeting components of epigenome by small molecules. Arch Pharm Res 37: 1367-1374, 2014.

11. Jones PA, Issa JP and Baylin S: Targeting the cancer epigenome for therapy. Nat Rev Genet 17: 630-641, 2016.

12. Ropero S and Esteller M: The role of histone deacetylases (HDACs) in human cancer. Mol Oncol 1: 19-25, 2007.

13. Li Y and Seto E: HDACs and HDAC inhibitors in cancer development and therapy. Cold Spring Harb Perspect Med 6: a026831, 2016.

14. Xu WS, Parmigiani RB and Marks PA: Histone deacetylase inhibitors: Molecular mechanisms of action. Oncogene 26: 5541-5552, 2007.

15. Minucci S and Pelicci PG: Histone deacetylase inhibitors and the promise of epigenetic (and more) treatments for cancer. Nat Rev Cancer 6: 38-51, 2006.

16. You JS, Kang JK, Lee EK, Lee JC, Lee SH, Jeon YJ, Koh DH, Ahn SH, Seo DW, Lee HY, et al: Histone deacetylase inhibitor apicidin downregulates DNA methyltransferase 1 expression and induces repressive histone modifications via recruitment of corepressor complex to pro-moter region in human cervix cancer cells. Oncogene 27: 1376-1386, 2008.

17. Gu W and Roeder RG: Activation of p53 sequence-specific DNA binding by acetylation of the p53 C-terminal domain. Cell 90: 595-606, 1997.

18. Zhao Y, Lu S, Wu L, Chai G, Wang H, Chen Y, Sun J, Yu Y, Zhou W, Zheng Q, et al: Acetylation of p53 at lysine 373/382 by the histone deacetylase inhibitor depsipeptide induces expression of p21(Waf1/Cip1). Mol Cell Biol 26: 2782-2790, 2006.

19. Evsyukova I, Bradrick SS, Gregory SG and Garcia-Blanco MA: Cleavage and polyadenyla-tion specificity factor 1 (CPSF1) regulates alternative splicing of interleukin 7 receptor (IL7R) exon 6. RNA 19: 103-115, 2013.

20. Jung M: Inhibitors of histone deacetylase as new anticancer agents. Curr Med Chem 8: 1505-1511, 2001. 
21. Goto H, Tomono Y, Ajiro K, Kosako H, Fujita M, Sakurai M, Okawa K, Iwamatsu A, Okigaki T, Takahashi T, et al: Identification of a novel phosphorylation site on histone $\mathrm{H} 3$ coupled with mi-totic chromosome condensation. J Biol Chem 274: 25543-25549, 1999.

22. Prigent $C$ and Dimitrov S: Phosphorylation of serine 10 in histone H3, what for? J Cell Sci 116: 3677-3685, 2003.

23. Kerdiles YM, Beisner DR, Tinoco R, Dejean AS, Castrillon DH DePinho RA and Hedrick SM: Foxol links homing and survival of naive T cells by regulating L-selectin, CCR7 and interleukin 7 receptor. Nat Immunol 10: 176-184, 2009.

24. Feng X, Wang H, Takata $\mathrm{H}$, Day TJ, Willen $\mathrm{J}$ and $\mathrm{Hu} \mathrm{H}$ Transcription factor Foxp1 exerts essential cell-intrinsic regulation of the quiescence of naive T cells. Nat Immunol 12: 544-550, 2011.

25. Goossens S, Radaelli E, Blanchet O, Durinck K, Van der Meulen J, Peirs S, Taghon T, Tremblay CS, Costa M,Farhang Ghahremani M, et al: ZEB2 drives immature T-cell lympho-blastic leukaemia development via enhanced tumour-initiating potential and IL-7 receptor sig-nalling. Nat Commun 6: 5794, 2015

26. Omilusik KD, Best JA, Yu B, Goossens S, Weidemann A, Nguyen JV, Seuntjens E, Stryjew-ska A, Zweier C, Roychoudhuri R, et al: Transcriptional repressor ZEB2 promotes terminal dif-ferentiation of $\mathrm{CD}^{+}$effector and memory $\mathrm{T}$ cell populations during infection. J Exp Med 212: 2027-2039, 2015.

27. Zhong C, Cui K, Wilhelm C, Hu G, Mao K, Belkaid Y, Zhao K and Zhu J: Group 3 innate lymphoid cells continuously require the transcription factor GATA-3 after commitment. Nat Im-munol 17: 169-178, 2016.

28. Daitoku H, Sakamaki J and Fukamizu A: Regulation of FoxO transcription factors by acety-lation and protein-protein interactions. Biochim Biophys Acta 1813: 1954-1960, 2011.

29. Garraway LA and Lander ES: Lessons from the cancer genome. Cell 153: 17-37, 2013.

30. Lawrence MS, Stojanov P, Mermel CH, Robinson JT, Garraway LA, Golub TR, Meyerson M, Gabriel SB, Lander ES and Getz G: Discovery and saturation analysis of cancer genes across 21 tumour types. Nature 505: 495-501, 2014.

31. Tran H, Brunet A, Griffith EC and Greenberg ME: The many forks in FOXO's road. Sci STKE 2003: RE5, 2003.
32. Accili D and Arden KC: FoxOs at the crossroads of cellular metabolism, differentiation, and transformation. Cell 117: 421-426, 2004.

33. Van Der Heide LP, Hoekman MF and Smidt MP: The ins and outs of FoxO shuttling: Mechanisms of FoxO translocation and transcriptional regulation. Biochem J 380: 297-309, 2004.

34. Barthel A, Schmoll D and Unterman TG: FoxO proteins in insulin action and metabolism. Trends Endocrinol Metab 16: 183-189, 2005

35. Calnan DR and Brunet A: The FoxO code. Oncogene 27: 2276-2288, 2008.

36. Nasrin N, Ogg S, Cahill CM, Biggs W, Nui S, Dore J, Calvo D, Shi Y, Ruvkun G and Alexan-der-Bridges MC: DAF-16 recruits the CREB-binding protein coactivator complex to the insulinlike growth factor binding protein 1 promoter in HepG 2 cells. Proc Natl Acad Sci USA 97: 10412-10417, 2000.

37. Essers MA, Weijzen S, de Vries-Smits AM, Saarloos I, de Ruiter ND, Bos JL and Burgering BM: FOXO transcription factor activation by oxidative stress mediated by the small GTPase Ral and JNK. EMBO J 23: 4802-4812, 2004.

38. Oh SW, Mukhopadhyay A, Svrzikapa N, Jiang F, Davis RJ and Tissenbaum HA: JNK regu-lates lifespan in Caenorhabditis elegans by modulating nuclear translocation of forkhead tran-scription factor/DAF-16. Proc Natl Acad Sci USA 102: 4494-4499, 2005.

39. Lehtinen MK, Yuan Z, Boag PR, Yang Y, Villén J, Becker EB, DiBacco S, de la Iglesia N, Gygi S, Blackwell TK, et al: A conserved MST-FOXO signaling pathway mediates oxidativestress responses and extends life span. Cell 125: 987-1001, 2006.

40. Kitamura YI, Kitamura T, Kruse JP, Raum JC, Stein R, Gu W and Accili D: FoxO1 protects against pancreatic beta cell failure through NeuroD and MafA induction. Cell Metab 2: 153-163, 2005.

41. Matsuzaki H, Daitoku H, Hatta M, Aoyama H, Yoshimochi K and Fukamizu A: Acetylation of Foxol alters its DNA-binding ability and sensitivity to phosphorylation. Proc Natl Acad Sci USA 102: 11278-11283, 2005. 